\title{
Convergence Analysis of an Optimal Scaling Algorithm for Semilinear Elliptic Boundary Value Problems
}

\author{
Goong Chen ${ }^{1, a}$, Berthold G. Englert ${ }^{2}$ and Jianxin Zhou ${ }^{1}$
}

\begin{abstract}
Proof of convergence for iterative schemes for finding unstable solutions of semilinear elliptic boundary value problems is an arduous task. In perspective is a special iterative algorithm using the idea of scaling. In the form called Scaling Iterative Algorithm (SIA) based on normalizing each iterate's function value to be 1 at a given interior point of the domain, it is found that SIA is computationally quite advantageous. Yet no convergence analysis is available. In this paper, we present a different idea of scaling which is an optimal scaling in the sense that the first integral is optimized. For this Optimal Scaling Iterative Algorithm (OSIA), we prove the convergence under certain assumptions on the nonlinearity and stipulated stepsize rule.
\end{abstract}

1: Department of Mathematics, Texas A\&M University, College Station, TX 77843.

E-mail addresses: gchen@math.tamu.edu and jzhou@math.tamu.edu.

2: Max Planck Institut für Quantanoptik, Garching, Germany.

E-mail address: bge@mpq.mpg.de.

3: Supported in part by a TITF initiative from Texas A\&M University. 


\section{Introduction}

In the computation of numerical solutions of semilinear elliptic boundary value problems (BVPs) in the form

$$
\left\{\begin{array}{l}
\Delta u(x)+f(x, u(x))=0, \quad x \in \Omega, \\
\left.B u\right|_{\partial \Omega}=0,
\end{array}\right.
$$

where in the above, $\Omega$ is a bounded open domain in $\mathbb{R}^{N}$ with sufficiently smooth boundary $\partial \Omega$, and $B$ is a linear boundary operator involving $u$ and $\frac{\partial u}{\partial n}$ (the normal derivative of $u$ on $\partial \Omega)$, several algorithms have been found to be effective ([4])

(1) DIA: the direct iteration algorithm;

(2) MIA: the monotone iteration algorithm;

(3) $M M A$ : the minimax algorithm, including the mountain-pass (MPA), the high-linking algorithm (HLA), etc.;

(4) SIA: the scaling iteration algorithm;

(5) Variational derivative iteration type algorithm such as Newton's algorithm or Sobolev gradients [16],

etc. Each algorithm has its advantages and disadvantages and should be chosen according to certain a priori knowledge of the solution manifold structure, if any such knowledge is available. For example, DIA and MIA are useful in capturing the so-called stable solutions (Morse index $=0$ ) while MPA and SIA are useful in capturing the so-called mountain pass solutions (Morse index=1) which are unstable. As for algorithm complexity, DIA, MIA and SIA are one-level algorithm and easy to program; MMA is a two-level algorithm which can be used to capture saddle points of high Morse index for a more general functional.

Associated with the various algorithms, whether theoretical or numerical, there is the all important question of convergence. Algorithms enable the finding of a solution of a nonlinear problem through iterations where at each iteration often an iterate of a linear problem is sought. Will the sequence or a subsequence thereof of iterates converge, in a certain sense, to a definitive solution of the given nonlinear problem? The answer of this question is well understood in the case of MIA, where the sequences of sub-solutions and super-solutions will sandwich a bona fide (stable) solution in the $C^{2, \alpha}(\Omega)$-norm $[1,18]$, under certain relatively easily verifiable) conditions on (1.1). However, for the various algorithmic versions of MPA and HLA ([4, 6, 7, 10] e.g.), proofs of convergence to an unstable solution turn out to be much more challenging. In the original formulation of the famous Mountain Pass Lemma by Ambrosetti and Rabinowitz [2], its proof is not totally constructive. Also compounding 
this difficulty is an associated variational problem which must be treated globally. For a theoretical method to be amenable to numerical implementation, adaptations are necessary. One of the main components of such adaptations is the localization of the global procedure in the Mountain Pass Lemma, resulting in several possible versions of MMA. Only recently, convergence analysis for a local MMA has been successfully carried out; see [13, 14, 15].

In this paper, our main interest is in SIA, the scaling iterative algorithm. The version of SIA as presented in [4, pp. 1573-1574] (see more details in (2.3)-(2.4) in Section 2) was found to be very effective in computing numerical solutions of a certain class of unstable solutions of semilinear elliptic BVPs, especially the Lane-Emden type equation whose nonlinearity is a power law. The accuracy and convergence rate of SIA in [4] totally agree with the MPA in every example computed in [4]. Yet SIA in $[4,5]$ has a major advantage over MPA in that SIA is an one-level algorithm and considerably easier to program and implement. However, the form of SIA as presented and used in [4] has not yet been placed a solid rigorous foundation because no convergence proof was established, despite many years of serious efforts by the authors of [4].

Recently, in a continuing attempt to analyze the convergence of SIA, we have come up with a new version of SIA which may be called an optimal scaling iteration algorithm (OSIA). The SIA in [4] was based on scaling at a single interior point of domain $\Omega$. However, the new OSIA is based on scaling along a given direction. It turns out to work much in the same way as in a theoretical study done much earlier by Ding and Ni [9]. This confluence of ideas is indeed a pleasure. Even though the work in [9] is not relevant to any iterative algorithms, credits are due to the authors of [9] for the priority of the intersected ideas. Here, our main interest lies in treating OSIA as an algorithm, with emphasis on its convergence analysis.

Because of the closeness and similarity, we are able to utilize the ideas of convergence analysis developed earlier by Li and Zhou [13]-[15] for MMA and applied them to OSIA. In Section 2, we describe the procedures of OSIA and relevant matters. In Section 3, convergence analysis of OSIA is given. Even though we must concede that convergence proof for SIA (as presented in [4]) is still open, from our work in Section 3 one can begin to understand why such a proof is challenging to clench. Hopefully, this paper will contain at least some more than tangential ideas and will also draw attention toward a convergence proof of SIA.

The various algorithms, DIA, MIA, MMA, SIA, Newton's, etc., may be implemented with finite difference (FDM), finite elements (FEM) and boundary elements (BEM). At the present time, error estimates for such algorithms and discretization schemes are available only for MIA; see [3, 8, 11, 12, 17], for example. This is a wide open area in numerical analysis of nonlinear PDEs where more investigations are most welcome. 


\section{Scaling Iteration Algorithms}

It is well-known that semilinear elliptic PDEs of the form

$$
\begin{cases}\Delta u-a u+b u^{p}=0 & \text { on } \Omega \\ u>0 & \text { on } \Omega \\ \left.u\right|_{\partial \Omega}=0, & \end{cases}
$$

where $a \geq 0$ and $b>0$ are two arbitrarily given constants and $p>1$, satisfy the "scaling property". For example, if $u$ is a solution of (2.1), then from $u$ we can find a solution $v$ to the problem

$$
\begin{cases}\Delta v-a v+b \lambda v^{p}=0 & \text { on } \Omega \\ v>0 & \text { on } \Omega \\ \left.v\right|_{\partial \Omega}=0 & \end{cases}
$$

for any constant $\lambda>0$ by taking $v=\lambda^{\frac{1}{1-p}} u$. This property can be exploited for computational purposes as follows. Consider solving (2.1). Define

$$
v(x)=\frac{u(x)}{u\left(x_{0}\right)}, \quad \text { for some fixed } \quad x_{0} \in \Omega
$$

Then $v$ satisfies

$$
\left\{\begin{array}{l}
\Delta v(x)-a v(x)+\lambda b v^{p}(x)=0, \Omega, \lambda \equiv\left[u\left(x_{0}\right)\right]^{p-1} \\
v\left(x_{0}\right)=1 \\
v>0, \Omega \\
\left.v\right|_{\partial \Omega}=0
\end{array}\right.
$$

We now define SIA as follows

\section{SIA}

Step 1: Choose any $v_{0}(x) \underset{\not \equiv}{\gtrless} 0$ on $\Omega ; v_{0}$ sufficiently smooth;

Step 2: Find $\lambda_{n+1} \geq 0$ and $v_{n+1}(\cdot)$ such that

$$
\left\{\begin{array}{l}
\Delta v_{n+1}(x)-a v_{n+1}(x)=-\lambda_{n+1} b v_{n}^{p}(x), \quad x \in \Omega \\
v_{n+1}\left(x_{0}\right)=1 \\
\left.v_{n+1}\right|_{\partial \Omega}=0
\end{array}\right.
$$

Step 3: If $\left\|v_{n+1}-v_{n}\right\|_{H_{0}^{1}(\Omega)}<\varepsilon$ output and stop. Else set $n=n+1$ and go to Step 2 .

Remark 2.1. It is clear that if in Step 3, we have $v_{k+1}-v_{k}=0$ then $v_{k+1}$ is a solution to (2.2) and consequently $u^{*}=\lambda_{n+1}^{\frac{1}{1-p}} v_{n+1}$ solves (2.1). Assume that DIA, the direct iteration algorithm, were applied to (2.1) as follows: with chosen $v_{0}(x) \underset{\not \equiv}{\gtrless}$ on $\Omega$, for $n=0,1,2, \ldots$, 
solve

$$
\left\{\begin{array}{l}
\Delta v_{n+1}-a v_{n+1}=-b v_{n}^{p} \text { on } \Omega, \\
\left.v_{n+1}\right|_{\partial \Omega}=0 .
\end{array}\right.
$$

Then what we have found numerically is that the above iteration converges either to the trivial solution $v_{\infty} \equiv 0$ or diverges to infinity. Thus the motivation for setting $v_{n+1}\left(x_{0}\right)=1$ in (2.3) is to prevent the convergence of $v_{\infty}$ to the trivial solution 0 , and to prevent $v_{n}$ from run away to infinity.

Before we present a variant of SIA, let us digress from iterative schemes for semilinear PDEs and instead look at the common Newton's algorithm for scalar-valued functions. This is intended to provide heuristics for the subsequent discussion. Recall Newton's method for solving a nonlinear equation $g(x)=0$ :

iteration: Take an initial guess $x_{0}$,

and then let

$$
x_{n+1}=x_{n}-\frac{g\left(x_{n}\right)}{g^{\prime}\left(x_{n}\right)} .
$$

See Fig. 1.

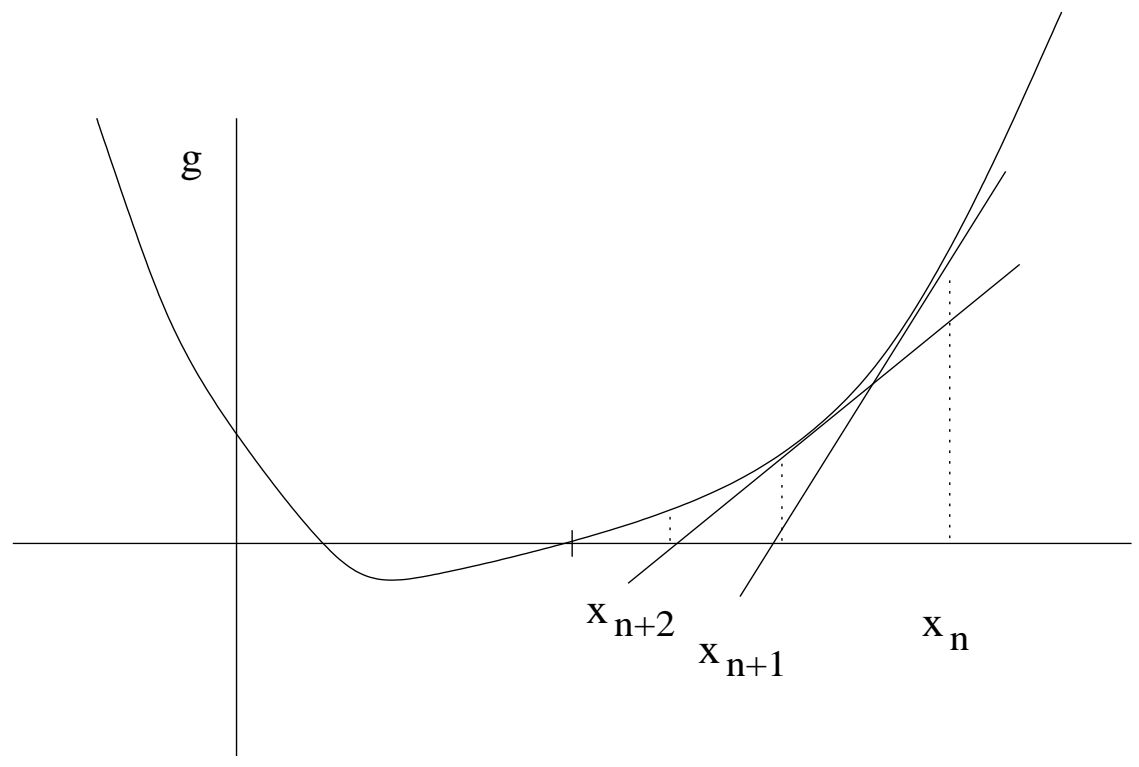

Figure 1: Newton's iteration to approximate a zero of a given function $g$.

Newton's method does not assume a variational structure (which actually should be utilized as much as possible if it is available). It may also fail to converge in the following situation; see Fig. 2. 


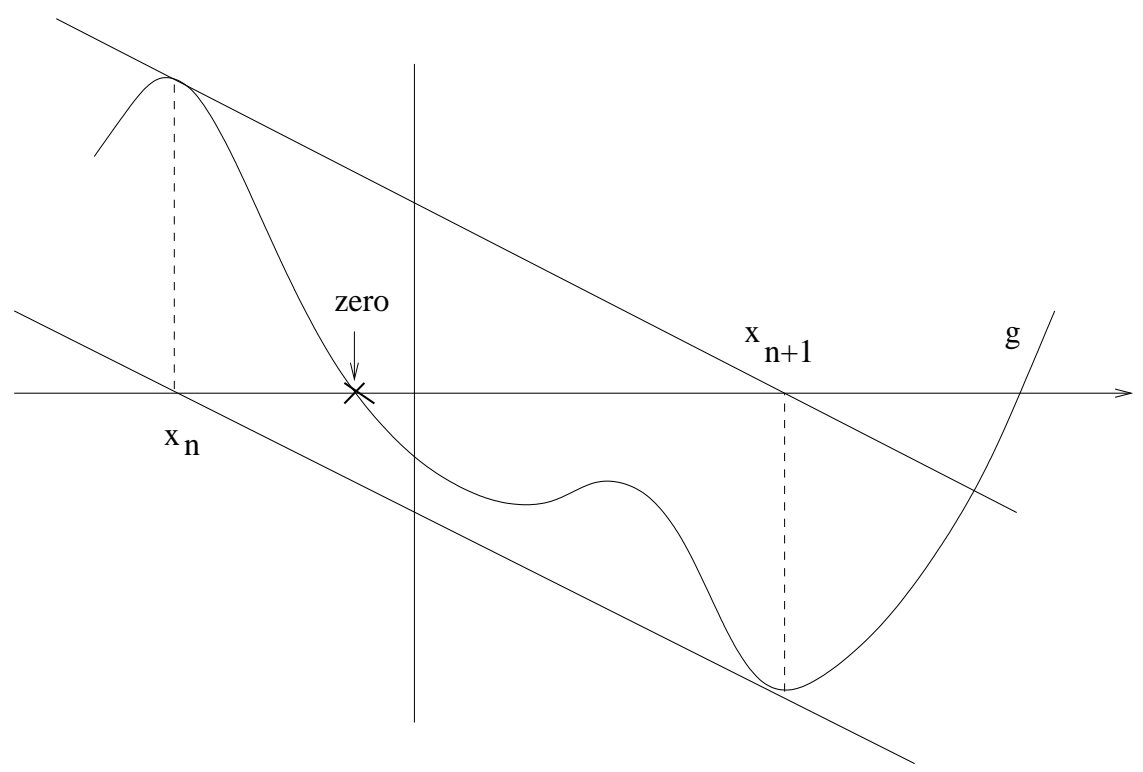

Figure 2: A situation where Newton's method fails to converge to a zero of $g$. The iteration repeats itself between $x_{n}$ and $x_{n+1}$.

The method is known to be unstable. Suppose that we want to find a critical point $u^{*}$ of a variational problem with first integral $J(v)$, i.e.,

$$
\text { find } u^{*} \text { s.t. } J^{\prime}\left(u^{*}\right)=0 \text {. }
$$

The infinite dimensional analogue of Newton' method becomes

$$
u_{n+1}=u_{n}-\left[J^{\prime \prime}\left(u_{n}\right)\right]^{-1} J^{\prime}\left(u_{n}\right) .
$$

It involves computation of the second derivative $J^{\prime \prime}\left(u_{n}\right)$ of an infinite-dimensional functional $J$ at $u_{n}$. Note that the iteration (2.6) could face serious trouble when $u^{*}$ satisfying (2.5) is a degenerate critical point, i.e., the operator

$$
J^{\prime \prime}\left(u^{*}\right) \text { does not exist or is not invertible. }
$$

In some sense, degeneracy is not avoidable in finding a saddle point. Let $u_{1}$ be a nondegenerate local minimum critical point of $J$. So $J^{\prime \prime}\left(u_{1}\right)$ has all positive eigenvalues. Let $u_{2}$ be a mountain pass solution (saddl epoint) to be found. We assume that $u_{2}$ is nondegenerate, $J^{\prime \prime}\left(u_{2}\right)$ has a negativeeigen value. If $J$ is $C^{2}$, then there will be a point $u_{0}$ between $u_{1}$ and $u_{2}$ such that $J^{\prime \prime}\left(u_{0}\right)$ has a zero eigenvalue. Thus $J^{\prime \prime}\left(u_{0}\right)$ is not invertible and around $u_{0}, J^{\prime \prime}$ is ill-conditioned. Such a case can be bypassed if an initial guess is chosen to be close to $u_{2}$. 
As to enhance stability of Newton's algorithm, there is some rescue from optimization theory. An important idea as suggested in Fig. 2 is to introduce a variable stepsize:

$$
u_{n+1}=u_{n}-s_{n}\left[J^{\prime \prime}\left(u_{n}\right)\right]^{-1} J^{\prime}\left(u_{n}\right),
$$

where $s_{n}$ satisfies certain stepsize rules (such as Armijo's [ ], Goldstein's [ ], or Powell's [ ], etc.). This modification facilitates convergence and enhances stability.

Now we are in a position to present an optimal scaling algorithm with variable stepsize, acronymed OSIA.

Consider the semilinear elliptic BVP

$$
\left\{\begin{array}{l}
\Delta u(x)+f(x, u(x))=0, \quad x \in \Omega \\
\left.u\right|_{\partial \Omega}=0
\end{array}\right.
$$

Let the corresponding first integral be

$$
J(u)=\int_{\Omega}\left[\frac{1}{2}|\nabla u|^{2}-F(x, u)\right] d x
$$

where $F$ satisfies

$$
\frac{\partial}{\partial u} F(x, u)=f(x, u)
$$

For the semilinear problem $(2.9)$, let $H=H_{0}^{1}(\Omega)$ and $\|\quad\|$ be the $H_{0}^{1}(\Omega)$-norm and denote $S=\{u \in H:\|u\|=1\}$. we now define the following iterative scheme:

\section{OSIA}

Step 1: Set $k=0$. Given $u_{k} \in S$.

Step 2: Find $\lambda_{k}=\arg _{\lambda} \max J\left(\lambda u_{k}\right)$ and set $v_{k}=\lambda_{k} u_{k}$.

Step 3: Solve $\bar{v}_{k}$ from

$$
\left\{\begin{array}{l}
-\Delta \bar{v}_{k}=f\left(\cdot, v_{k}\right), \quad \text { on } \Omega \\
\left.\bar{v}_{k}\right|_{\partial \Omega}=0
\end{array}\right.
$$

Step 4: If $\left\|\bar{v}_{k}-v_{k}\right\|<\varepsilon$ then stop and exit, else go to Step 5 .

Step 5: Denote

$$
u_{k}(s)=\frac{s \bar{v}_{k}+(1-s) v_{k}}{\left\|s \bar{v}_{k}+(1-s) v_{k}\right\|} \in S, 0<s \leq 1 \quad \text { and } \quad \lambda_{k}(s)=\arg \max _{\lambda} J\left(\lambda u_{k}(s)\right)
$$

choose $0<s_{k} \leq 1$ satisfying certain stepsize rule, e.g., (2.11).

Step 6: Set $v_{k+1}=\lambda_{k}\left(s_{k}\right) u_{k}\left(s_{k}\right)$ and update $k=k+1$, then go to Step 3 .

Remark 2.2. Note that the problem settings for both SIA and OSIA assume a variational structure. But SIA does not utilize its variational structure, while Steps 2 and 5 in OSIA use its variational structure. It is clear that the problem setting (2.1) of OSIA is more general 
than the problem setting (2.9) of SIA. If in Step 4 of OSIA, we have $\bar{v}_{k}-v_{k}=0$, then $\bar{v}_{k}$ is a solution to (2.9). The normalization in Step 5 of OSIA signifies "return to the unit sphere $S^{\prime \prime}$.

There are several different ways to define a stepsize rule, e.g., we may define

Definition 2.1. Let $v_{k}, \bar{v}_{k}, \lambda_{k}, \lambda_{k}(s)$ and $u_{k}(s)$ be defined as in OSIA. If $\bar{v}_{k} \neq v_{k}$ then we define the stepsize rule by

$$
s_{k}=\max \left\{0<s \leq 1 \mid J\left(\lambda_{k}(s) u_{k}(s)\right)-J\left(v_{k}\right) \leq-\frac{1}{2} \frac{s \lambda_{k}}{\left\|v_{k}+s\left(\bar{v}_{k}-v_{k}\right)\right\|}\left\|\bar{v}_{k}-v_{k}\right\|^{2}\right\} .
$$

Example 2.1. A model problem is the Lane-Emden equation

$$
\begin{cases}\Delta u(x)+u^{p}(x)=0 & \text { on } \Omega \\ u(x)>0 & \text { on } \Omega, \\ \left.u\right|_{\partial \Omega}=0 . & \end{cases}
$$

The first integral is

$$
J(v)=\int_{\Omega}\left[\frac{1}{2}|\nabla v|^{2}-\frac{1}{p+1}|v|^{p+1}\right] d x .
$$

The key iteration step in Steps 3 says that $\bar{v}_{k}$ is determined from solving

$$
-\Delta \bar{v}_{k}=\lambda_{k}^{p} u_{k}^{p}
$$

where $\lambda_{k}$ is chosen such that $J\left(\lambda u_{k}\right)$ is maximized at $\lambda=\lambda_{k}$. Thus

$$
\frac{d}{d \lambda} \int_{\Omega}\left[\frac{1}{2} \lambda^{2}\left|\nabla u_{k}\right|^{2}-\frac{1}{p+1} \lambda^{p+1}\left|u_{k}\right|^{p+1}\right] d x=0,
$$

i.e.,

$$
\lambda_{k}=\left[\int_{\Omega}\left|\nabla u_{k}\right|^{2} d x / \int_{\Omega}\left|u_{k}\right|^{p+1} d x\right]^{1 / p-1} .
$$

Therefore, the scaling in (2.13) is "optimal" in this sense.

\section{Convergence of OSIA}

With the variational functional $J$ in $(2.10)$, under some standard conditions on $f$, a direct computation shows that (weak) solutions to (2.9) coincide with critical points of $J$. We now proceed to prove the convergence of OSIA under certain assumptions on the nonlinearity $f$ and the stipulated stepsize rule (2.11). 
Let $\langle$,$\rangle denote the inner product in H_{0}^{1}(\Omega)$ and let $d=-J^{\prime}(u)$ be the negative gradient of $J$ at $u$. For each $v \in H_{0}^{1}(\Omega)$, we have

$$
\langle d, v\rangle=\left\langle-J^{\prime}(u), v\right\rangle \equiv-\left.\frac{d}{d t} J(u+t v)\right|_{t=0}
$$

Then

$$
\int \nabla d \cdot \nabla v d x=\int_{\Omega}[-\nabla u \cdot \nabla v+f(\cdot, u) v] d x
$$

or

$$
-\int \Delta d \cdot v d x=\int_{\Omega}[\Delta u+f(\cdot, u)] v d x
$$

Thus $d=-J^{\prime}(u)$ solves

$$
\left\{\begin{array}{l}
\Delta d=-[\Delta u+f(\cdot, u)] \text { on } \Omega \\
\left.d\right|_{\partial \Omega}=0
\end{array}\right.
$$

Note that if

$$
\left\{\begin{array}{l}
-\Delta \bar{v}_{k}=f\left(\cdot, v_{k}\right) \text { on } \Omega \\
\left.\bar{v}_{k}\right|_{\partial \Omega}=0
\end{array}\right.
$$

then

$$
\left\{\begin{array}{l}
-\Delta\left(\bar{v}_{k}-v_{k}\right)=\Delta v_{k}+f\left(\cdot, v_{k}\right) \text { on } \Omega, \\
\left.\left(\bar{v}_{k}-v_{k}\right)\right|_{\partial \Omega}=0
\end{array}\right.
$$

or $d_{k} \equiv \bar{v}_{k}-v_{k}=-J^{\prime}\left(v_{k}\right)$ is the negative gradient of $J$ at $v_{k}$. Now Step 4 in OSIA reads:

$$
\text { If }\left\|d_{k}\right\|<\varepsilon \text { then stop and exit, else got to Step } 3 \text {. }
$$

OSIA becomes a critical point approximation algorithm. To prove its convergence, let us rewrite $u_{k}(s)$ in Step 5 of OSIA as

$$
u_{k}(s)=\frac{v_{k}+s\left(\bar{v}_{k}-v_{k}\right)}{\left\|v_{k}+s\left(\bar{v}_{k}-v_{k}\right)\right\|}=\frac{v_{k}+s d_{k}}{\left\|v_{k}+s d_{k}\right\|}=\frac{u_{k}+s_{v} d_{k}}{\left\|u_{k}+s_{v} d_{k}\right\|}
$$

where $s_{v}=\frac{s}{\left\|v_{k}\right\|}$. The stepsize rule (2.11) becomes

$$
s_{k}=\max \left\{0<s \leq 1 \mid J\left(\lambda_{k}(s) u_{k}(s)\right)-J\left(v_{k}\right) \leq-\frac{1}{2} \frac{s \lambda_{k}}{\left\|v_{k}+s d_{k}\right\|}\left\|d_{k}\right\|^{2}\right\} .
$$

With $H=H_{0}^{1}(\Omega)$ and $S=\{u \in H:\|u\|=1\}$, let $J$ be given in (2.10). For each $u \in S$, define the peak selection of $J$ by $p(u)=\lambda_{u} u$ where $\lambda_{u}=\arg \max _{\lambda} J(\lambda u)$. It is clear that OSIA becomes a variant of the local minimax method presented in [13]. Thus in general, OSIA is a two-level algorithm. Howver when an explicit formula for computing $\lambda_{k}$ is available, such as in Example 2.1, OSIA remains an one-level algorithm. The relation 
between the negative gradient and the steepest descent direction can be seen as follows. The steepest descent direction of $J$ at a point $u$ is a unit vector $d_{u}$ such that

$$
d_{u}=\arg \max _{d \in H,\|d\|=1}\left\langle-J^{\prime}(u), d\right\rangle
$$

When $H$ is a Hilbert space, the right hand side of (3.3) is equal to $\left\|-J^{\prime}(u)\right\|$. Thus $d_{u}=\frac{-J^{\prime}(u)}{\left\|J^{\prime}(u)\right\|}$ is the steepest descent direction which differs from the negative gradient only by a positive factor. Note that we are looking for a critical point $u^{*}$, i.e., $J^{\prime}\left(u^{*}\right)=0$. Since dividing by a term going to zero will introduce an extra error term in numerical computation and there is a stepsize before the steepest descent direction in the algorithm, this stepsize can absorb a positve factor anyway, we can simply use the negative gradient to replace the steepest descent direction in the algorithm.

Following similar arguments as in $[13,14]$, we can prove

Theorem 3.1. (Theorem 4.3 in [13]) Assume that Conditions (h1) - (h5') in [13] are satisfied and that there exist $a_{5}>0$ and $a_{6}>0$ s.t. for $s$ as specified in (h2),

$$
\left|f_{\xi}(x, \xi)\right| \leq a_{5}+a_{6}|\xi|^{s-1} .
$$

Then the peak selection $p$ of $J$ is $C^{1}$.

Now we assume that Conditions (h1) - (h5') in [13] and (3.4) hold.

Theorem 3.2. ([13, 14]) Let $u^{k}$ and $v^{k}=p\left(u^{k}\right), k=0,1,2, \ldots$ be the sequence generated by OSIA, then

(a) $J\left(v_{k+1}\right)<J\left(v_{k}\right)$, i.e., OSIA is a strictly decreasing algorithm and thus stable;

(b) $\left\{v^{k}\right\}_{k=0}^{\infty}$ has a subsequence $\left\{v^{k_{i}}=p\left(u^{k_{i}}\right)\right\}$ that converges to a critical point;

(c) any limit point of $\left\{v^{k}\right\}$ is a critical point of J. In particular, any convergent subsequence $\left\{v^{k_{i}}\right\}$ of $\left\{v^{k}\right\}$ converges to a critical point.

If a critical point is not isolated, we can only expect some point-to-set convergnce as similar to Theorem 3.3 in [14]. When a critical point is isolated, by a similar proof of Theorem 3.4 in [14], we can establish the following point-to-point convergence.

Theorem 3.3. If $J(p(\bar{u}))=\operatorname{loc} \min _{u \in S} J(p(u))$ and $\bar{v}=p(\bar{u}) \neq 0$ is an isolated critical point, then there exists an open set $U$ in $H$, such that $\bar{u} \in U \cap S$ and starting from any $u_{0} \in U \cap S$, the sequence $\left\{v^{k}\right\}_{k=0}^{\infty}$ generated by OSIA converges to $\bar{v}=p(\bar{u})$.

Theorem 3.4. (Theorem 3.3 in [15]) Assume that Conditions (h1) - (h5') in [13] and (3.4) are satisfied, then a critical point found by OSIA has Morse index 1. 


\section{Numerical Examples}

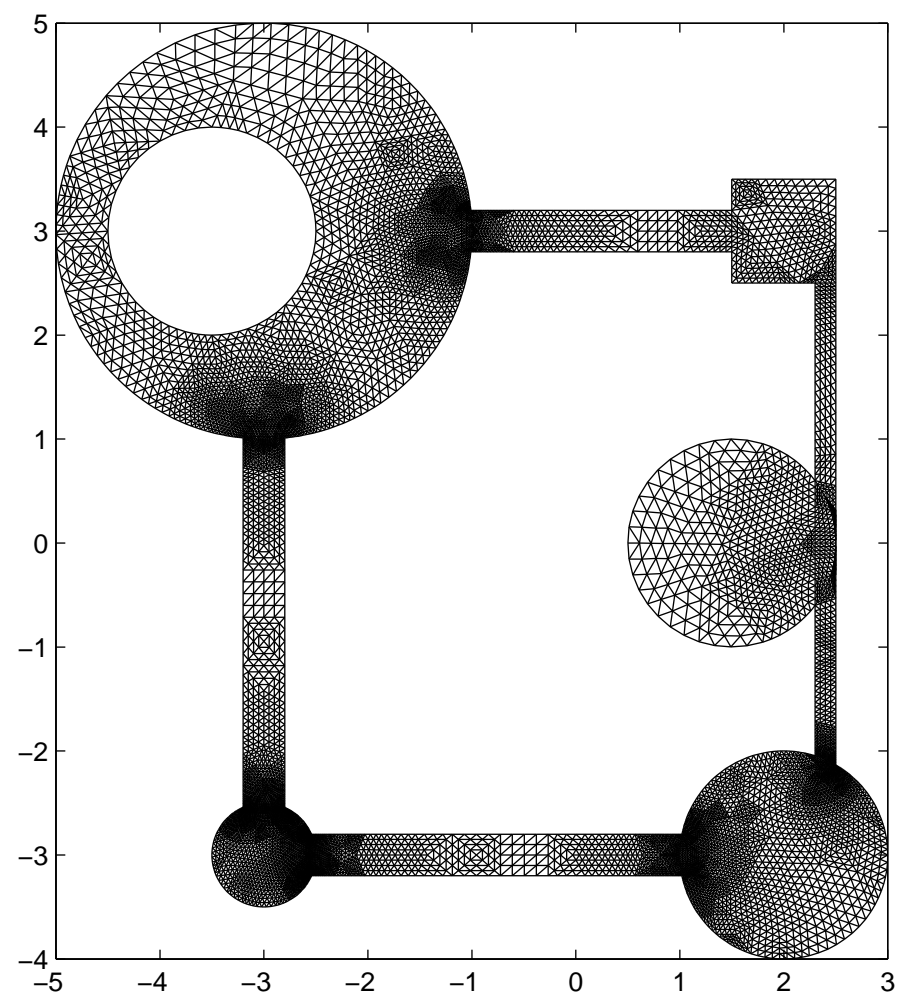

Figure 3: The domain and its totally 10321 triangular finite elements. 

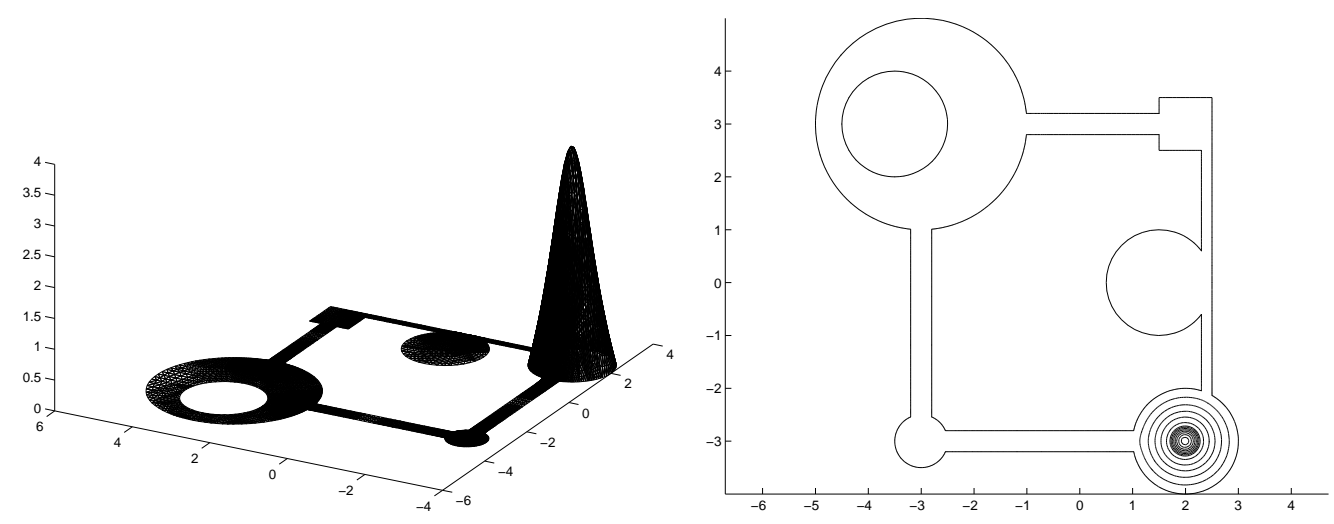

Figure 4: A solution to () with $J=10.9576$
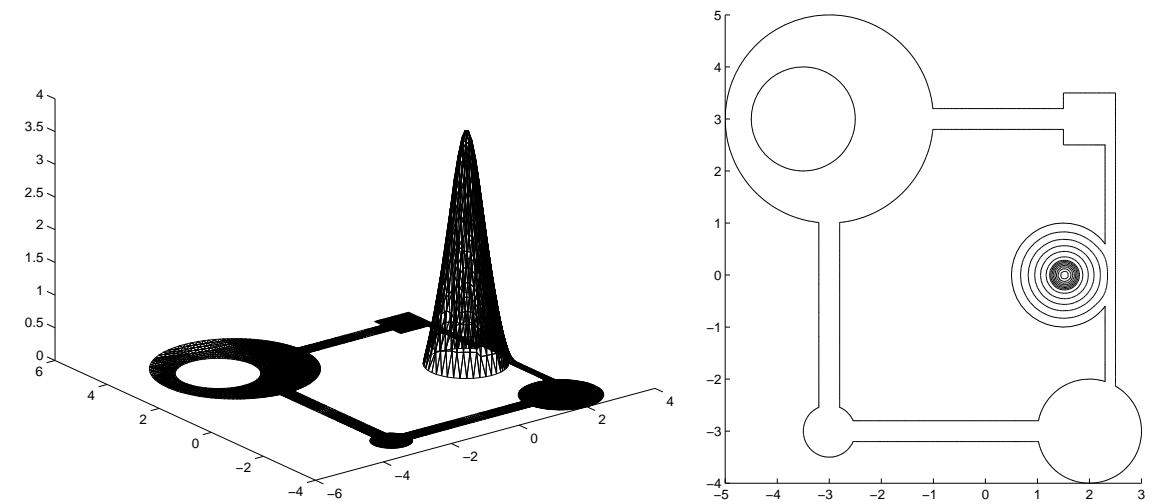

Figure 5: A solution to () with $J=11.0232$
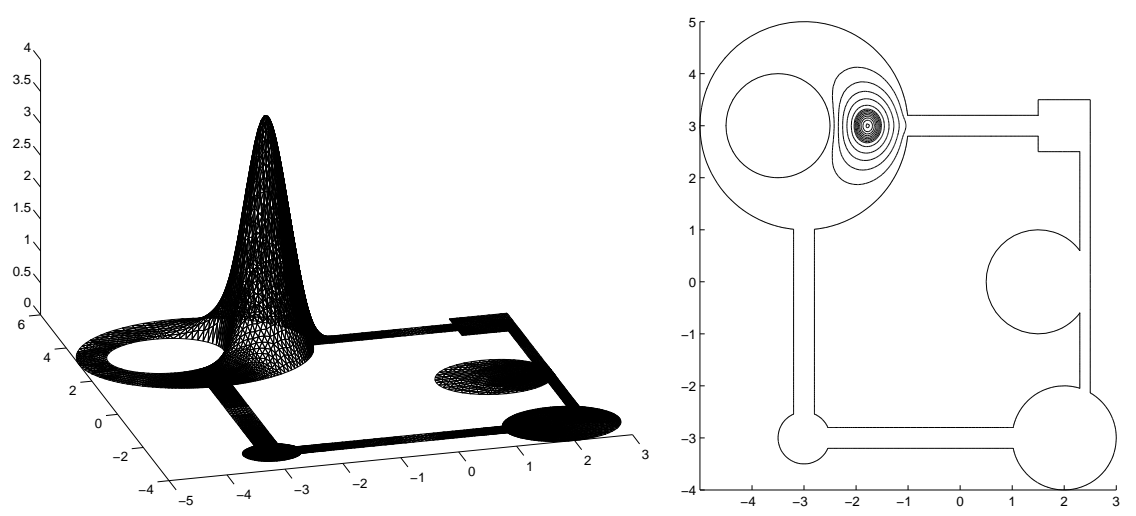

Figure 6: A solution to () with $J=11.6854$ 

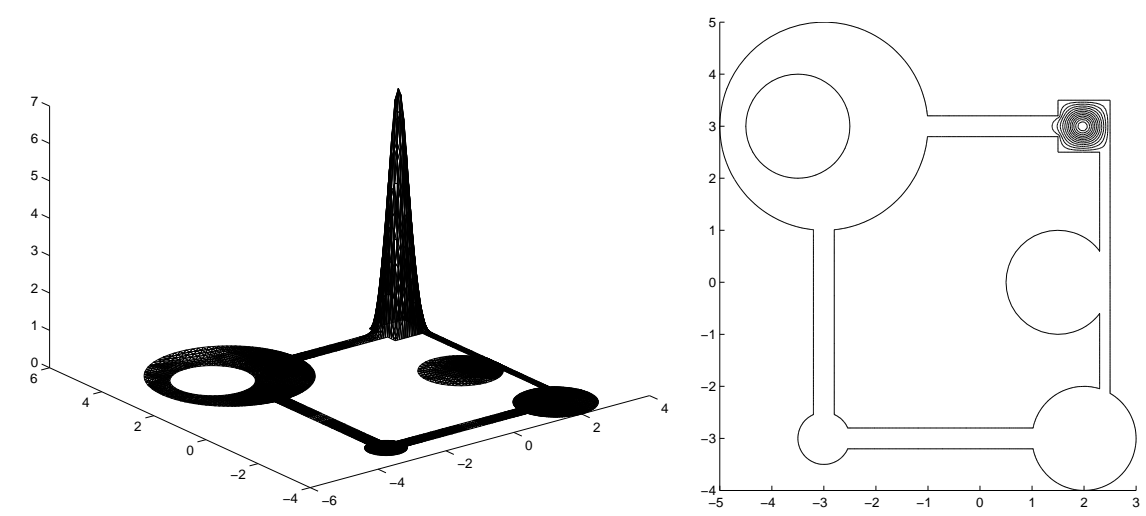

Figure 7: A solution to () with $J=37.07$
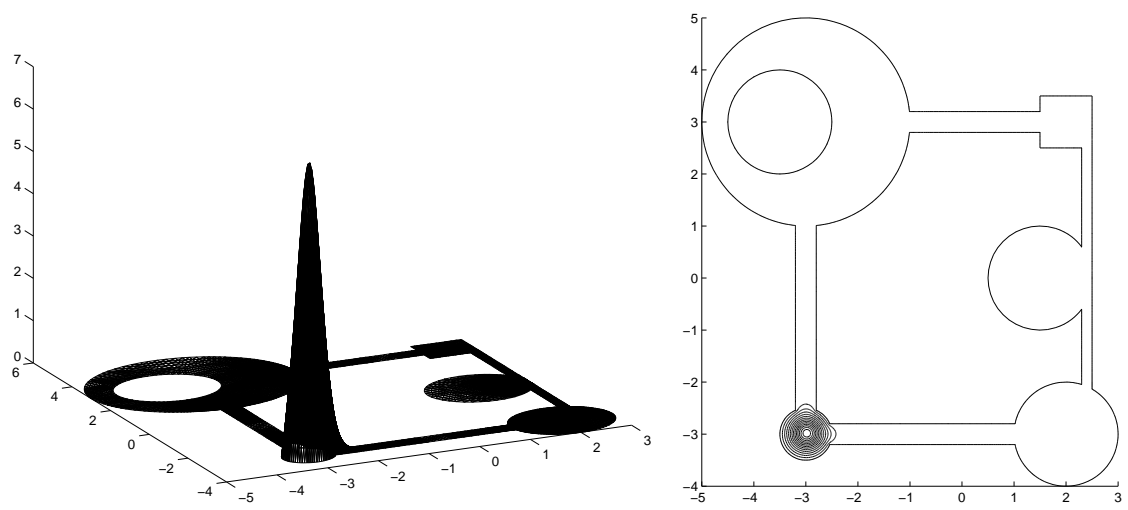

Figure 8: A solution to () with $J=41.4318$
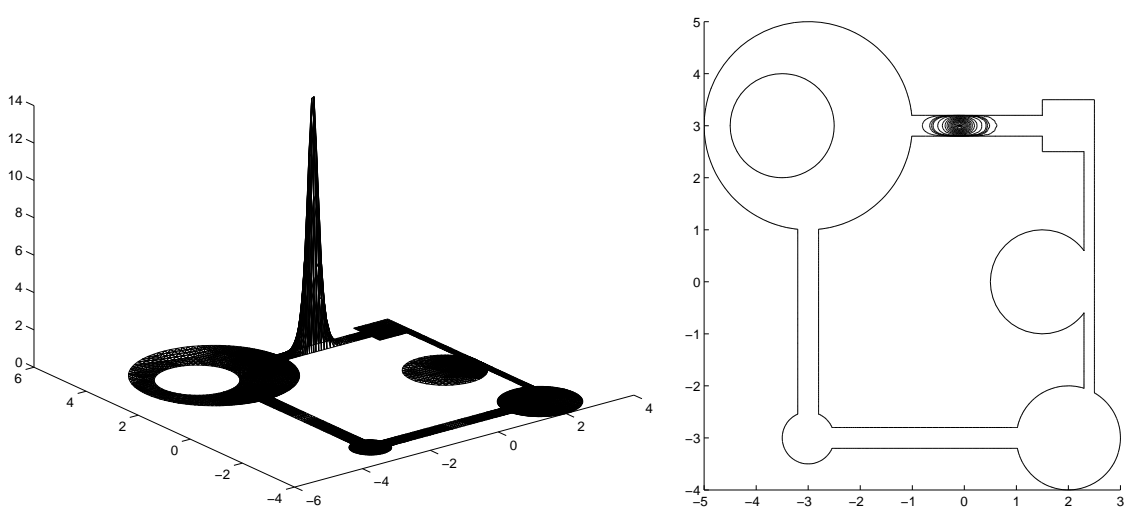

Figure 9: A solution to () with $J=179.9271$ 

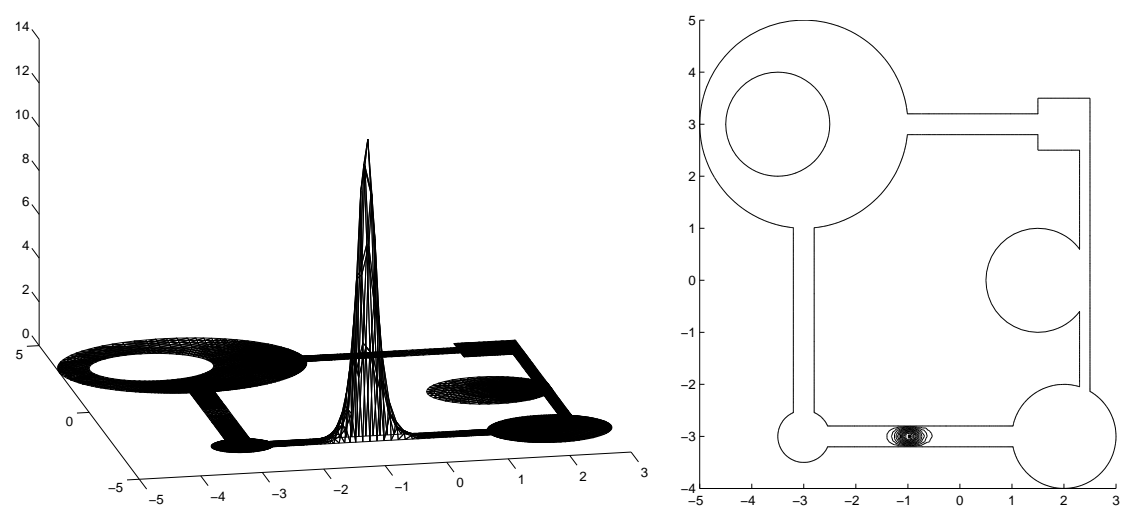

Figure 10: A solution to () with $J=195.9461$
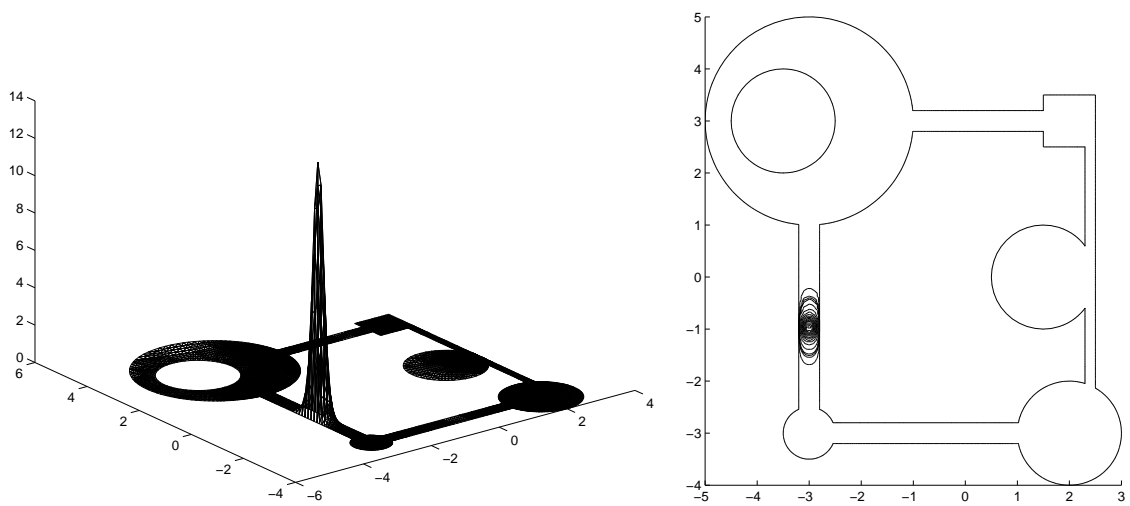

Figure 11: A solution to () with $J=195.954$
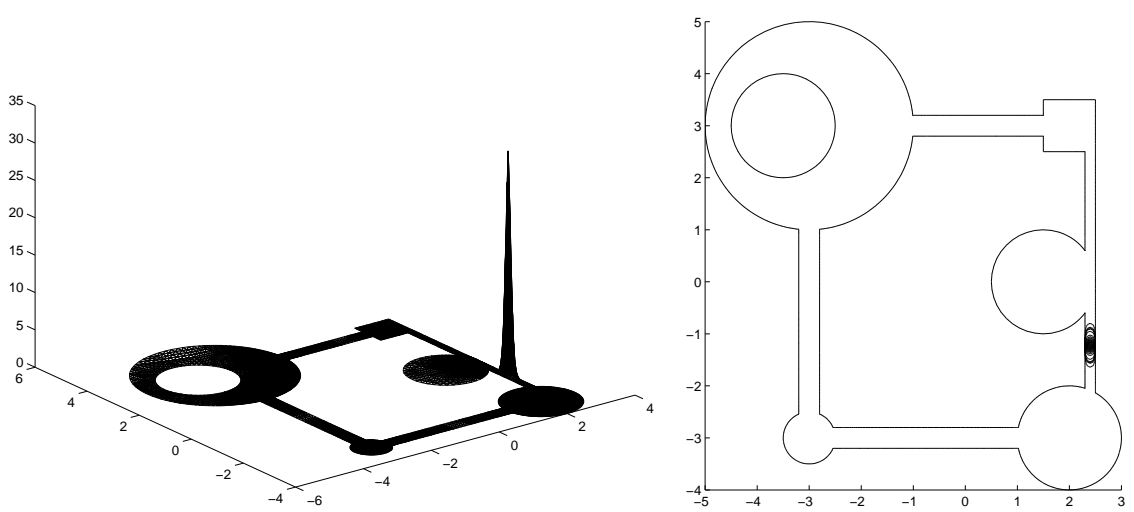

Figure 12: A solution to () with $J=865.4277$ 

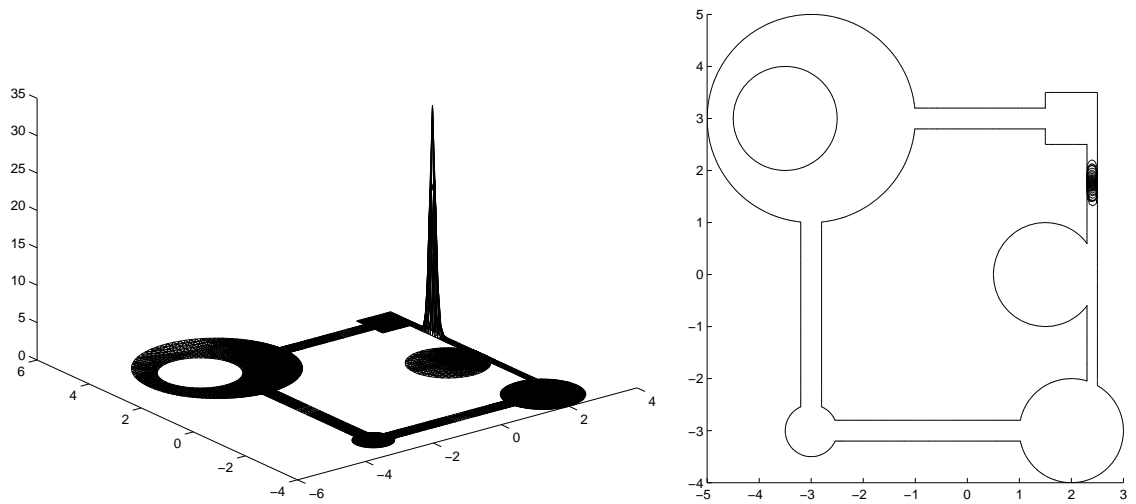

Figure 13: A solution to () with $J=971.1523$

\section{References}

[1] H. Amann, Supersolution, monotone iteration and stability, J. Diff. Eq 21 (1976), 367377.

[2] A. Ambrosetti and P.H. Rabinowitz, Dual variational methods in critical point theory and applications, J. Funct. Anal. 14 (1973), 349-381.

[3] G. Chen, Y. Deng, W.M. Ni and J. Zhou, Boundary element monotone iteration scheme for semilinear elliptic partial differential equations, Part II: Quasimonotone iteration for coupled $2 \times 2$ systems, Math. Comp. 69 (1999), 629-652.

[4] G. Chen, W.M. Ni and J. Zhou, Algorithms and visualization for solutions of nonlinear elliptic equations, Int. J. Bifurc. \& Chaos 7 (2000), 1565-1612.

[5] G. Chen, W.M. Ni, A. Perronnet and J. Zhou, ibid, Part II: Dirichlet, Neumann and Robin boundary conditions and problems in 3D, Int. J. Bifurc. Es Chaos 11 (2001), 1781-1799.

[6] Y.S. Choi and P.J. McKenna, A mountain pass method for the numerical solution of semilinear elliptic problems, Nonlin. Anal. 20 (1993), 417-437.

[7] Y.S. Choi and P.J. McKenna, Numerical mountain pass methods for nonlinear boundary value problems, in "Nonlinear Problems in Applied Mathematics", T.S. Angell, et al., eds., SIAM, Philadelphia, 1996, 86-95.

[8] Y. Deng, G. Chen, W.M. Ni and J. Zhou, Boundary element monotone iteration scheme for semilinear elliptic partial differential equations, Math. Comp. 65 (1996), 943-982. 
[9] W.Y. Ding and W.M. Ni, On the existence of positive entire solutions of a semilinear elliptic equation, Arch. Rat. Mech. Anal. 91 (1986), 283-308.

[10] Z. Ding, D. Costa and G. Chen, A high linking method for sign changing solutions of semilinear elliptic equations, Nonlin. Anal. 38 (1999), 151-172.

[11] C.U. Huy, P.J. McKenna and W. Walter, Finite difference approximations to the Dirichlet problem for elliptic systems, Numer. Math. 49 (1986), 227-237.

[12] K. Ishihara, Monotone explicit iterations of the finite element approximations for the nonlinear boundary value problems, Numer. Math. 45 (1984), 419-437.

[13] Y. Li and J. Zhou, Local characterizations of saddle points and their Morse indices, in "Control Nonlinear Distributed Parameter Systems", G. Chen, I. Lasiecka and J. Zhou, eds., Chap. 11, Lecture Notes in Pure \& Appl. Math., Vol. 218, Marcel Dekker, New York, 2001, 233-251.

[14] Y. Li and J. Zhou, A minimax method for finding multiple critical points and its applications to semilinear PDE, SIAM J. Sci. Comp. 23 (2001), 840-865.

[15] Y. Li and J. Zhou, Convergence results of a local minimax method for finding multiple critical points, to appear in SIAM J. Sci. Comp.

[16] J.W. Neuberger, Sobolev Gradients and Differential Equations, Lecture Notes in Mathematics, Vol. 1670, Springer, Berlin-Heidelberg-New York, 1997.

[17] C.V. Pao, Block monotone iterative methods for numerical solutions of nonlinear elliptic equations, Numer. Math. 72 (1995), 239-262.

[18] D. Sattinger, Monotone methods in nonlinear elliptic and parabolic boundary value problems, Indiana Univ. Math. J. 21 (1971/72), 979-1000. 